\title{
Mapping residual stress profiles at the micron scale using FIB micro- hole drilling
}

\author{
B. Winiarski ${ }^{a}$ and P. J. Withers ${ }^{b}$
}

\author{
The University of Manchester, School of Materials, Grosvenor St, Manchester, M1 7HS, UK \\ âB.Winiarski@manchester.ac.uk, ${ }^{\mathrm{b} P}$ hilip.Withers@manchester.ac.uk
}

Keywords: residual stress, micron-scale, FIB, digital image correlation, hole drilling.

\begin{abstract}
Measuring residual stress at the sub-micron scale imposes experimental challenges. We propose a new technique, namely the incremental micro-hole-drilling method (I $\mu \mathrm{HM})$, for measurement of residual stress profiles as a function of depth with high spatial definition. Like its macroscale counterpart, it is applicable to either crystalline or amorphous materials, but at the submicron scale. Our method involves micro-hole milling using the focused ion beam of a dual beam FEGSEM/FIB microscope. The surface displacements are tracked by digital image correlation of SEM images recorded during milling. The displacement fields mapped around the whole are used to reconstruct the variation of the in-plane stress tensor as a function of depth. In this way the multiaxial state of residual stress has been characterised around drilled holes of 2 microns or so, enabling the profiling of the stress variation at the sub-micron scale to a depth of 2 microns. Here we demonstrate the efficacy of this method by measuring the stresses in a surface-severe-plasticallydeformed ( $\left.\mathrm{S}^{2} \mathrm{PD}\right) \mathrm{Zr}_{50} \mathrm{Cu}_{40} \mathrm{Al}_{10}$ bulk metallic glass (in atomic percent, at.\%) sample after failure under four-point-bending-fatigue.
\end{abstract}

\section{Introduction}

Residual stresses exist in most materials across a range of scales [1, 2]. They arise as a consequence of prior processing and/or in-service loading. Reliable information about the state of residual stress in a component or structure is often an essential part of the structural integrity assessment. At the macro scale the Incremental Hole Drilling Method [3-6] is a well established semi-destructive method for measurement of non-uniform residual stresses near the surface of a component. In essence, the method involves drilling a small shallow hole to a depth not greater than its diameter (a few millimetres say) in the specimen. The strain introduced by the partial relaxation of the stress around the hole is recorded by a strain gauge rosette stepwise over a series of drilling increments. Subsequently, the residual stress profiles are back-calculated using the Integral Method [4, 5] based on calibration data calculated by finite elements. The tendency for unstable residual stress solutions to the Integral Method, when small increments of hole-depth are selected either at very shallow or at depths approaching the hole diameter, is countered by a Tikhonov regularization scheme [7].

Destructive and semi-destructive techniques based on mechanical relaxation phenomena, such as hole/core drilling [4, 5, 8], slitting [9], and curvature methods [10] can be applied to both crystalline and amorphous materials. In principle they can be scaled down and applied to smaller structures than those to which they have been applied to-date. The advent of dual beam focused ion beam field emission gun scanning electron microscopes (FIB-FEGSEM) in combination with digital image correlation analysis (DIC) has made it possible to make very fine holes or excisions and to record the resulting displacements with high precision. This has recently led to a number of microscale analogues of the mechanical stress measurement methods, principally using cantilevers or slots [11-15]. The disadvantage of these techniques is that they tend to measure one component of the in-plane stress tensor and have relatively poor lateral spatial resolution (tens of microns).

In this paper we outline the measurement principle of a new FIB micro-hole drilling method based on the Incremental Hole Drilling Method [4, 5]. We adopt a Tikhonov regularization scheme 
[7] to stabilise the reconstructed residual stress profiles $\left(\sigma_{x}, \sigma_{y}\right.$ and $\left.\tau_{x y}\right)$. Here this new method is used to drill a $4 \mu \mathrm{m}$ hole to estimate the residual-stresses tensor with a depth resolution of $\sim 200 \mathrm{~nm}$ and a lateral resolution of around $10 \mu \mathrm{m}$ in a surface-severe-plastically-deformed (S $\left.{ }^{2} \mathrm{PD}\right)$ $\mathrm{Zr}_{50} \mathrm{Cu}_{40} \mathrm{Al}_{10} \mathrm{BMG}$ (in at.\%) system after failure under four-point-bending-fatigue [15].

\section{Experimental Description}

Material preparation. $\mathrm{Zr}, \mathrm{Cu}$ and $\mathrm{Al}$ melts ${ }^{\dagger}$ were argon arc-melted and tilt-casted to obtain $\mathrm{Zr}_{50} \mathrm{Cu}_{40} \mathrm{Al}_{10}$ (atm\%) BMG. Then one side of the specimen was argon shot-peened with WC/Co shots. Later the fatigue behaviour of the BMG was studied under four-point-bending-fatigue [15].

Surface Contrast Enhancement for DIC. The reliability of the DIC analysis and the scalability of the FIB-based hole drilling method strongly depend on the size, distribution, and density of the surface features that are tracked [16]. Here we have decorated the specimen surface with 20 to $30 \mathrm{~nm}$ equiaxed yttria-stabilized zirconia (YSZ) particles $(\sim 13 \%$ coverage) precipitated from an ethanol suspension (see Fig. 1a). We used an ultrasonic bath to break up large YSZ particle conglomerates in the suspension prior to application. To minimise surface charging and to 'protect' the surface from $\mathrm{Ga}^{+}$implantation, the surface of the specimen is coated with a carbon film of $220 \AA$ thickness using a Gatan PECS 682 etching-coating system equipped with a Gatan 681.20000 Film Thickness Monitor.
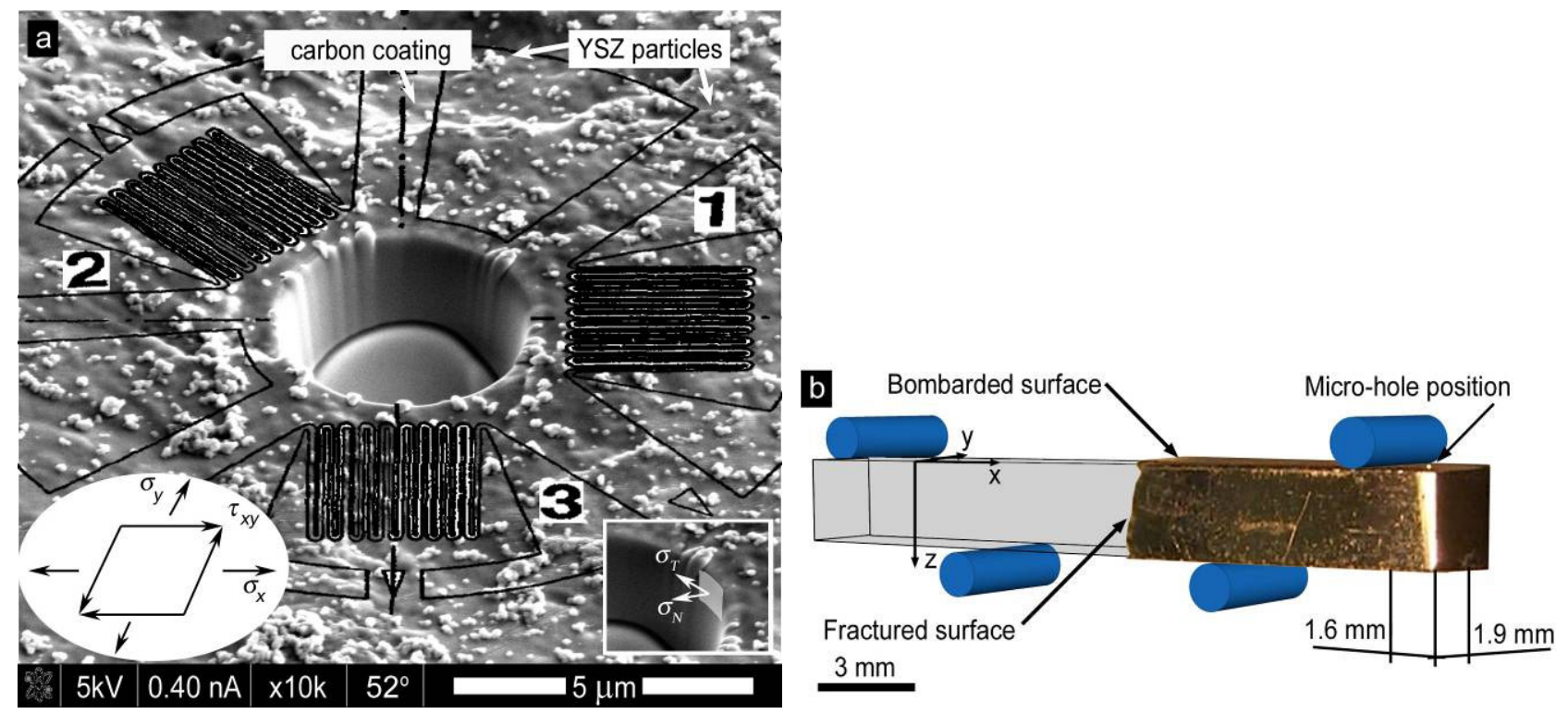

Figure 1. FEGSEM image of the microhole of radius $r_{a}=4 \pm 0.07 \mu \mathrm{m}$ and depth, $z=1.8 \pm 0.07 \mu \mathrm{m}$ : (a) showing the micro-hole at an oblique viewing angle with superimposed virtual strain-gauge rosette $r_{a} / r=0.50$, where $\mathrm{r}$ is the radius of strain-gauge rosette; the surface is decorated with YSZ nano-particles and then carbon coated (the film thickness is about $20 \mathrm{~nm}$ ); (b) general view showing fatigue test configuration and location of the micro-hole.

FIB Micro-hole Drilling. In order to map the stress profile in a severely plastically peen deformed BMG at the location shown in Fig. $1 \mathrm{~b}$, we have introduced a micro-hole of $2 \mu$ m radius to a depth of $1.8 \mu \mathrm{m}$ (see Fig. 1a) in 11 increments using FEI Dual Beam xT Nova NanoLab 600i FEGSEM/FIB microscope. This was achieved using a focused $\mathrm{Ga}^{+}$ion beam of $280 \mathrm{nA}$ accelerated by an electric field of $30 \mathrm{kV}$. At each increment the stress relaxation was recorded by FEGSEM image taken normal to the surface at a resolution of $1024 \times 884$ pixels taken with magnification of $10,000 \times$. A focused electron beam of $0.40 \mathrm{nA}$ was accelerated by an electric field of $5 \mathrm{kV}$ and secondary electrons were detected using Everhart Thornley Detector (ETD). Each FEGSEM image was an average from 8 scans with a dwell time of the electron beam of $3 \mu \mathrm{s}$.

\footnotetext{
${ }^{\dagger}$ purity better then $99.9 \%$ by weight
} 
Digital Image Correlation Analysis. The surface displacements due to the stress relaxations are mapped from FEGSEM images at each increment using LaVision DaVis 7.2 DIC software (see Figure 2a). The DIC analysis is undertaken at locations corresponding to the positions of strain gauges of a virtual strain-gauge rosette of $r_{a} / r=0.50$, where $r_{a}$ is the radius of the hole and $r$ is the radius of the strain gauge rosette, see Figs $1 \mathrm{a}$ and $2 \mathrm{~b}$. During the analysis, images are divided into smaller sub-regions (patches), which are individually correlated [16]. Before analyzing the microhole milling experiment, the best vector calculation parameters (VCP) were determined from two FEGSEM images captured before hole milling as described elsewhere [15]. Estimated DIC analysis accuracy is presented in Table 1. The random error is determined as the standard deviation of strain measurement determined from two FEGSEM images captured before the hole milling. Whereas the systematic error is the scatter in strain measurement using different VCPs: $32 \times 32$ pixels overlapped by 25 and $50 \%$, and $64 \times 64$ pixels overlapped by 50 and $75 \%$.

Table 1. Estimated DIC uncertainty for a single vector.

\begin{tabular}{|c|c|c|}
\hline Component & Random error, $\varepsilon_{r n d}[\mu \varepsilon]$ & Systematic error, $\varepsilon_{s y s}$ \\
\hline$\varepsilon_{G 1}, \varepsilon_{G 2}, \varepsilon_{G 3}$, & 330 & $\pm 8 \%$ of measured value \\
\hline
\end{tabular}
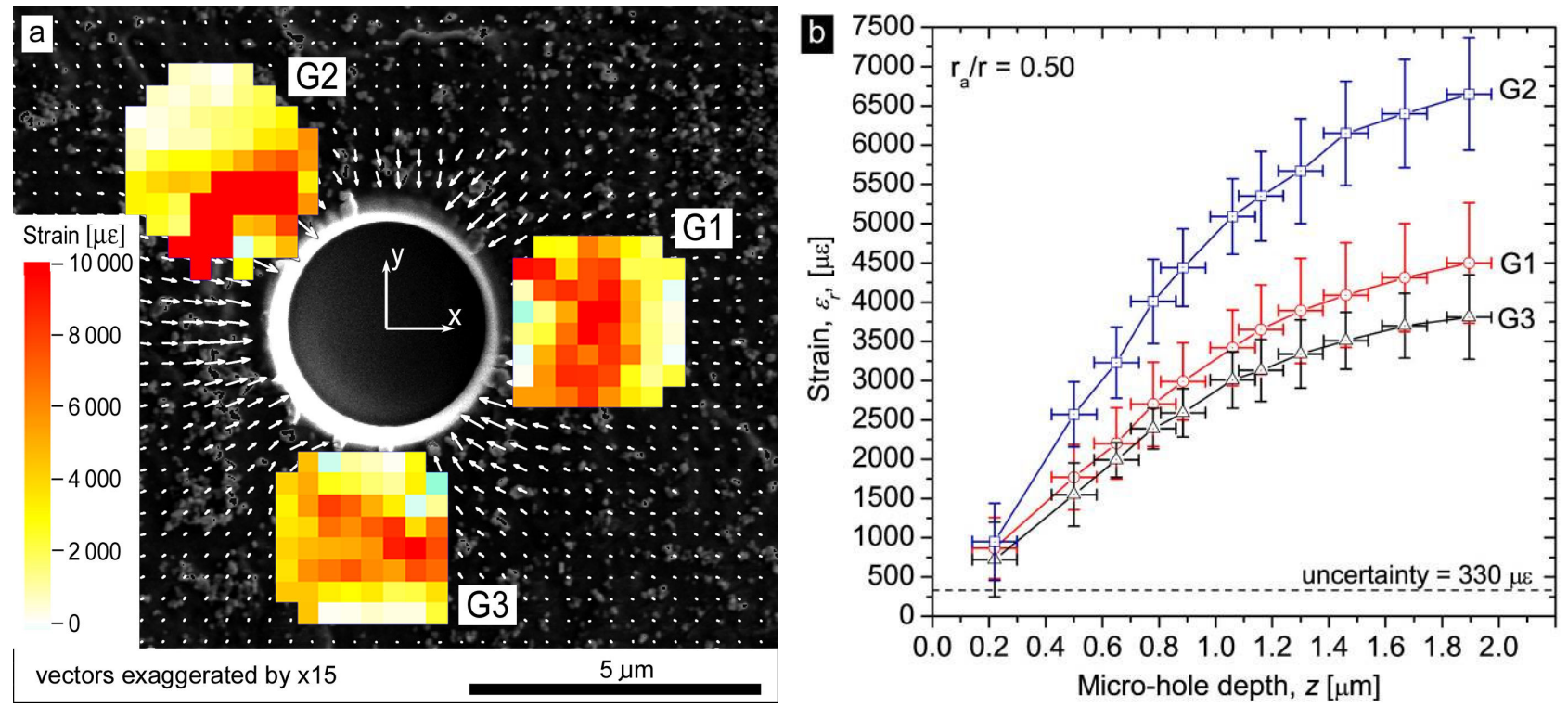

Figure 2. Digital image correlation analysis results for the final increment, $z=1.8 \pm 0.07 \mu \mathrm{m}$ : (a) 2-D displacement vector field (vectors are magnified $\times 15$ ) with the radial strains measured at the locations corresponding to a virtual strain-gauge rosette of $r_{a} / r=0.50$ superimposed; (b) average radial strains, $G 1, G 2$ and $G 3$, vs. hole depth, $z$.

The Integral Method. To infer the residual stress profiles $\left(\sigma_{x}, \sigma_{y}\right.$ and $\left.\tau_{x y}\right)$ with depth we used directly the Integral Method proposed by Schajer [4, 5]. In essence the non-uniform stress profiles can be calculated using three pseudo-inversion equations in matrix notation

$$
\begin{aligned}
& {[P]=E /(1+v)\left[\bar{a}^{T} \bar{a}\right]^{-1}[\bar{a}]^{T}[p],} \\
& {[Q]=E\left[\bar{b}^{T} \bar{b}\right]^{-1}[\bar{b}]^{T}[q],} \\
& {[T]=E\left[\bar{b}^{T} \bar{b}\right]^{-1}[\bar{b}]^{T}[t],}
\end{aligned}
$$

where $E=95 \pm 5 \mathrm{GPa}$, and $v=0.37[17] ;[\bar{a}]$ and $[\bar{b}]$ are lower triangular matrices of coefficients corresponding to the cumulative strain relaxation functions for hole drilling into a equibiaxial and pure shear stress field, respectively. We assembled these matrices using the bivariate interpolation function and tabulated cumulative strain relaxation functions from [5]. The transformed stress variables (the column matrices $P, Q$ and $T$ ) in Eqs. (1) - (3) which are acting at corresponding holedepths in a plane parallel to the specimen surface are related to the Cartesian stress components as

$$
[P]=\left[\left(\sigma_{y}+\sigma_{x}\right) / 2\right]
$$




$$
\begin{aligned}
& {[Q]=\left[\left(\sigma_{y}-\sigma_{x}\right) / 2\right],} \\
& {[T]=\left[\tau_{x y}\right] .}
\end{aligned}
$$

And the three strain relaxations measured at each hole-depth (the column matrices $p, q$ and $t$ ) in Eqs. (1) - (3) are expressed in terms of transformed strain variables: $[p]=\left[\left(\varepsilon_{G 3}+\varepsilon_{G 1}\right) / 2\right]$, $[q]=\left\lfloor\left(\varepsilon_{G 3}-\varepsilon_{G 1}\right) / 2\right\rfloor$ and $[t]=\left\lfloor\left(\varepsilon_{G 1}+\varepsilon_{G 3}-2 \varepsilon_{G 2}\right) / 2\right\rfloor$. The Cartesian stress components can be calculated from Eqs. (4) - (6)

Stabilisation of Reconstruction Algorithm. The pseudo-inversion algorithms, Eqs. (1) - (3), yield stable residual stress solutions if the entries of coefficients matrices $[\bar{a}]$ and $[\bar{b}]$ are of the same order of magnitude. However, the micro-hole milling process produces relatively small matrix entries in the diagonal bands of the coefficient matrices. Thus, Eqs. (1) - (3), become numerically ill-conditioned leading to unstable residual stress solutions. In practice this results in large oscillations of the residual stress solution with depth (see Fig. 3a). The problem of the illconditioned incremental calibration function matrices has been widely investigated [18]. In essence, the schemes proposed to counter this are based on careful selection of the hole-depths at which the residual stresses are calculated, which usually lead to in-depth stress profiles inferred using a reduced number of data points. In order to overcome the numerical ill-conditioning and to stabilise the residual stress solutions we used the Tikhonov regularisation scheme proposed by Tjhung \& Li [7]. This scheme is significantly less sensitive to the depth increments chosen to back-calculate the residual stress profiles. We regularised Eqs. (1) - (3) into following form

$$
\begin{aligned}
& {\left[P_{\alpha}\right]=E /(1+v)\left[\alpha H_{2}^{T} H_{2}+\bar{a}^{T} \bar{a}\right]^{-1}[\bar{a}]^{T}[p],} \\
& {\left[Q_{\alpha}\right]=E\left[\alpha H_{2}^{T} H_{2}+\bar{b}^{T} \bar{b}\right]^{-1}[\bar{b}]^{T}[q],} \\
& {\left[T_{\alpha}\right]=E\left[\alpha H_{2}^{T} H_{2}+\bar{b}^{T} \bar{b}\right]^{-1}[\bar{b}]^{T}[t],}
\end{aligned}
$$

where $\alpha$ is the regularisation parameter and $H_{2}$ is the second difference matrix operator. Within the adopted regularisation scheme we select the regularisation parameter basing on an estimate $\delta$ of the error in the measured data, e.g. $[p]$. The parameter $\alpha$ is chosen a posteriori in such way that the Euclidean norm of regularised solution discrepancy, e.g. $\left\|(1+v) / E \cdot[\bar{a}]\left[P_{\alpha}\right]-[p]\right\|$ is equal to $\delta$ : the details are presented elsewhere [7]. The discrepancy in the measured displacements $\delta_{\text {est }}=\left\|\left[\varepsilon_{r d n}\right]+\left[\varepsilon_{s y s}\right]\right\|$, the least-squares solution discrepancy $\delta_{0}=\|(1+v) / E \cdot[\bar{a}][P]-[p]\|$, and the discrepancy after regularization $\delta$ are shown in Table 2.

Table 2. The discrepancy for the measurement after regularisation

\begin{tabular}{|c|c|c|}
\hline$\delta_{\text {est }}[\mu \varepsilon]$ & $\delta_{0}[\mu \varepsilon]$ & $\delta[\mu \varepsilon]$ \\
\hline 818 & 719 & 963 \\
\hline
\end{tabular}

Stress Calculation Error. Several sources of measurement error arise leading to errors of different types [19]. For the I $\mu \mathrm{HM}$ method the errors originate from five main sources: (a) strain measurement errors, which include DIC calculation errors, material redeposition and additional residual stresses induced by $\mathrm{Ga}^{+}$ions implantation; (b) hole depth measurement errors, which include non-flatness of the bottom of the hole; (c) hole diameter measurement errors, which include tapering of the hole and deviation from roundness; (d) incorrect material constants; (e) hole eccentricity, which include possible focused ion beam drifts and the effects of possible eccentricity of DIC strain-gauge rosette from the centre of the micro-hole. In this study, following the argument in [19], we include the source (a) where the strain perturbations, e.g. [ $\delta p]$ (the random error and the systematic error) result in calculated residual stress perturbation, e.g. $[\delta P]$, in Eqs. (1) - (3) and Eqs. (9) - (11). 
Application of the $\mathbf{I} \boldsymbol{\mu H M}$ method. Figures $3 \mathrm{a}$ and $\mathrm{b}$ shows the local in-plane residual stress profiles within the immediate vicinity of the surface of the $\mathrm{S}^{2} \mathrm{PD}$ treated $\mathrm{Zr}_{50} \mathrm{Cu}_{40} \mathrm{Al}_{10}$ bulk metallic glass as a function of depth. The same sample has been studied previously over a larger depth range in [15]. Since we used a relatively large number of data points, the matrices $[\bar{a}]$ and $[\bar{b}]$ in Eqs. (1) - (3) are ill-conditioned, which result in large oscillations of the residual stress solution with depth and extremely large uncertainty levels, in Fig. 3a. The regularization procedure has effectively reduced the oscillations of the residual stress solution, simultaneously keeping the uncertainties at acceptable level, in Fig. 3b. Our results indicate that compressive residual stress component, $\sigma_{x}$, in the longitudinal direction of the sample (see Figure 1c) appears to be somewhat higher $(<15 \%)$ than the stress component, $\sigma_{y}$, in the transverse direction. This difference may arise as a result geometry of the peened bar. There is some evidence of shear stresses $(\sim 230 \mathrm{MPa})$ indicating that the principal stresses lie at around $20^{\circ}$ to the specimen length. The longitudinal stress averages to $-920 \mathrm{MPa}$ over the evaluation depth $(1.8 \mu \mathrm{m})$ and compares to $-650 \mathrm{MPa}$ obtained using the FIB microslotting method to a depth of $4.1 \mu \mathrm{m}$ [15]. Some scatter in the data could arise from material redeposition in the vicinity of the micro-hole, which is more intense than for FIB microslotting method. The virtual strain gauge rosette method uses the displacement vectors only within 3 patches; we have found that this method tends to overestimate by about $10 \%$ the inferred residual stress profiles when compared with the quasi full-field method that uses all the vectors in an annulus around the hole [20].
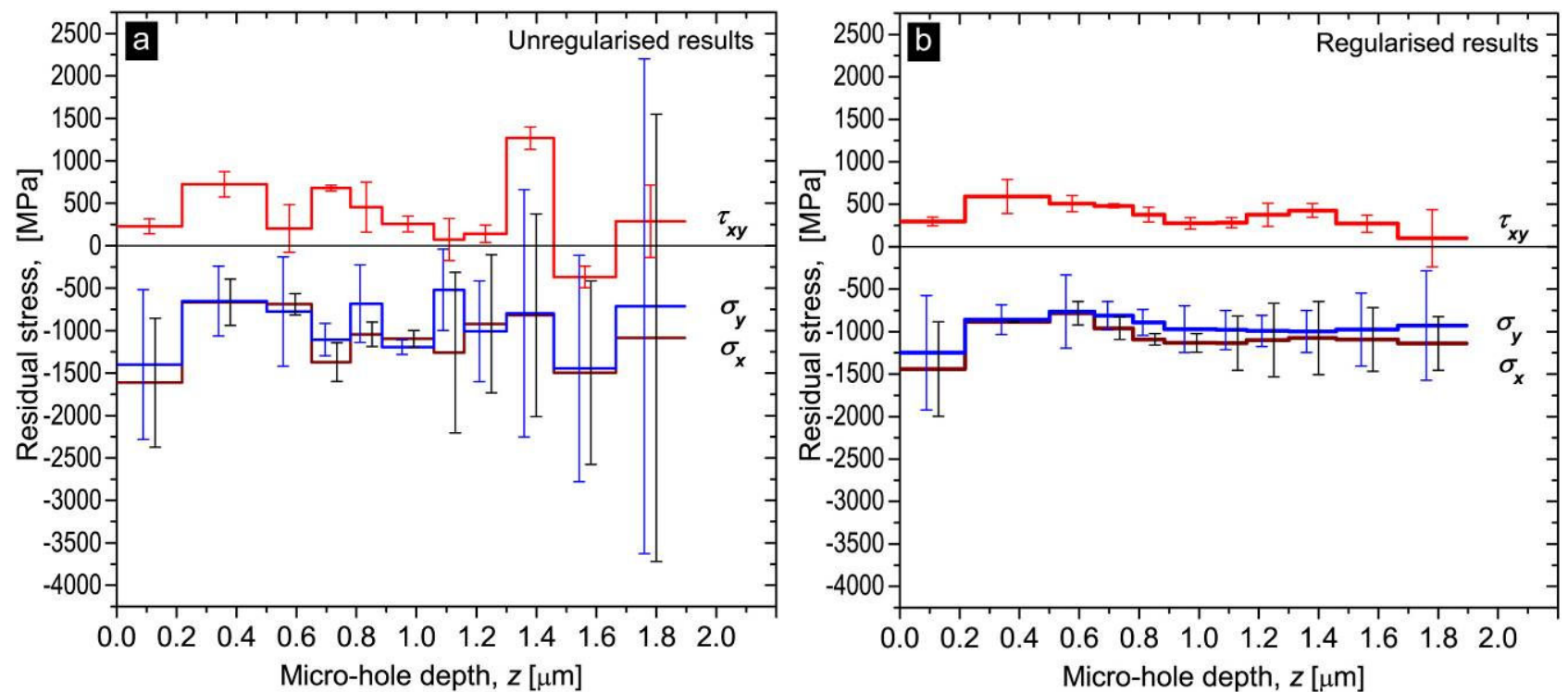

Figure 3. Inferred in-plane residual stresses profiles as a function of depth from the deformed surface: (a) unregularised stress components $\sigma_{x}, \sigma_{y}$ and $\tau_{x y}$; (b) regularised stress components $\sigma_{x}, \sigma_{y}$ and $\tau_{x y}$.

Conclusions. In summary, this short paper presents a new method based on the integral method for mapping in-plane residual or applied stresses as a function of depth at the micron-scale laterally and sub-micron scale depth-wise. Instability problems associated with the Integral Method, when small increments of hole-depth are selected near or far from the surface were minimised by application of a Tikhonov regularization scheme [7]. The results obtained by the new method reasonably agree with the results inferred by other relaxation methods [15]. The potential applications of this technique are wide-ranging including stresses in amorphous thin films, MEMS components and devices, organic electronic devices, nanostructured materials, etc. Though applicable to crystalline materials, for amorphous materials our micro-hole milling method has few competitors.

Acknowledgments. The stress measurements were made within the Stress and Damage Characterization Unit at the University of Manchester supported by the Light Alloys Towards Environmentally Sustainable Transport (LATEST) EPSRC Portfolio Project. We are grateful to P. Liaw and Y. Yokoyama for provision of the BMG. 


\section{References}

[1] P.J. Withers: Reports On Progress In Physics Vol. 70(12) (2007), p. 2211-2264.

[2] I.C. Noyan and P.J. Withers: Stresses in Microelectronic Circuits, in Encyclopedia of Materials: Science \& Technology, K.H.J. Buschow, Cahn, R.W., Flemings, M.C., et al., Editor. Elsevier: Oxford (2001).

[3] G.S. Schajer: Experimental Mechanics (2009), DOI: 10.1007/s11340-009-9228-7.

[4] G.S. Schajer: Journal Of Engineering Materials And Technology-Transactions Of The Asme Vol. 110(4)(1988), p. 338-343.

[5] G.S. Schajer: Journal Of Engineering Materials And Technology-Transactions Of The Asme Vol. 110(4)(1988), p. 344-349.

[6] P.V. Grant, J.D. Lord and P.S. Whitehead: The Measurement fo Residual Stresses by the Incremental Hole Drilling Technique, in Measurement Good Practice Guide, National Physical Laboratory: Teddington (2002).

[7] T. Tjhung and K.Y. Li: Journal Of Engineering Materials And Technology-Transactions Of The Asme Vol. 125(2)(2003), p. 153-162.

[8] McGinnis, M.J., S. Pessiki, and H. Turker: Experimental Mechanics Vol. 45(4)(2005), p. 359-367.

[9] G.S. Schajer and M.B. Prime: Journal Of Engineering Materials And TechnologyTransactions Of The Asme Vol. 129(2)(2007), p. 227-232.

[10] C.A. Klein: Journal Of Applied Physics, Vol. 88(9)(2000), p. 5487-5489.

[11] K.J. Kang, N. Yao, M.Y. He and A.G. Evans: Thin Solid Films Vol. 443(2003), p. 71-77.

[12] N. Sabate, D. Vogel, A. Gollhardt, J. Keller, C. Cané, I. Gràcia, J.R. Morante and B. Michel: Journal Of Micromechanics And Microengineering Vol. 16(2)(2006), p. 254-259.

[13] J. McCarthy, Z. Pei, M. Becker and D. Atteridge: Thin Solid Films Vol. 2000 (1-2)(2000), p. 146-151.

[14] S. Mass1, J. Keckes, and R. Pippan: Scripta Materialia Vol. 59(5)(2008), p. 503-506.

[15] B. Winiarski, R.M. Langford, J. Tian, Y. Yokoyama, P.K. Liaw and P.J. Withers: Metallurgical and Materials Transactions A - In Proof (2009).

[16] J. Quinta De Fonseca, P.M. Mummery and P.J. Withers: Journal of Microscopy Vol. 218(2004), p. 9-21.

[17] J.M. Pelletier, Y. Yokoyama and A. Inoue: Materials Transactions, Vol. 47(2007), p. 13591362.

[18] J.V. Beck, B. Blackwell and C.R. St.Clair Jr.: Inverse Heat Conduction - Ill-Posed Problems ( New York: Wiley-Interscience 1985).

[19] Schajer, G.S. and E. Altus: Journal Of Engineering Materials And Technology-Transactions Of The Asme Vol. 118(1)(1996), p. 120-126.

[20] B. Winiarski and P.J. Withers: Mapping of stress at the sub-micron scale by incremental focused ion beam hole-milling. Paper submitted 